\title{
Regulation of the expression of tumor necrosis factor-related genes by abnormal histone H3K27 acetylation: Implications for neural tube defects
}

\author{
CHUNLEI WAN, XIAOZHEN LIU, BAOLING BAI, HAIYAN CAO, HUILI LI and QIN ZHANG \\ Beijing Municipal Key Laboratory of Child Development and Nutriomics, Capital Institute of Pediatrics, \\ Peking University Teaching Hospital, Beijing 100020, P.R. China
}

Received November 4, 2016; Accepted June 20, 2017

DOI: $10.3892 / \mathrm{mmr} .2018 .8900$

\begin{abstract}
The association between apoptosis and neural tube defects (NTDs) is recognized as important, however, the precise link remains to be elucidated. Epigenetic modifications in human NTDs have been detected previously. In the present study, the occurrence of epigenetic modifications in apoptosis-related genes was investigated in a retinoic acid (RA)-induced mouse NTD model. Among 84 key genes involved in programmed cell death, 13 genes, including tumor necrosis factor (Tnf), annexin A5, apoptosis inhibitor 5, Bcl2-associated athanogene 3, baculoviral IAP repeat-containing 3, caspase (Casp)12, Casp4, Casp8, lymphotoxin $\beta$ receptor, NLR family, apoptosis inhibitory protein 2 , TNF receptor superfamily (Tnfrsf) 1a, TNF superfamily (Tnfs) $f 10$ and Tnfsf12, were downregulated, whereas nucleolar protein 3 was upregulated in the RA-induced NTD mice. Chromatin immunoprecipitation assays revealed that the regulatory regions of these differentially expressed TNF-related genes showed reduced histone H3K27 acetylation in NTDs, compared with control mice without NTDs. Reverse transcription-quantitative polymerase chain reaction revealed that H3K27ac-binding to the differentially regulated genes was markedly decreased in the NTD mice, whereas binding to the unchanged genes Casp3 and Nfkbl was unaffected. In conclusion, certain TNF-related genes appeared to be downregulated in NTDs, possibly as a result of abnormal histone H3K27 acetylation. These results shed new light on the epigenetic dysregulation of apoptosis-related genes in NTDs.
\end{abstract}

Correspondence to: Dr Qin Zhang or Dr Huili Li, Beijing Municipal Key Laboratory of Child Development and Nutriomics, Capital Institute of Pediatrics, Peking University Teaching Hospital, 2 Yabao Road, Chaoyang, Beijing 100020, P.R. China

E-mail: maureenzq@hotmail.com

E-mail: lihuili2011@gmail.com

Key words: retinoic acid, apoptosis, tumor necrosis factor-related genes, H3K27 acetylation, neural tube defects

\section{Introduction}

Neural tube defects (NTDs) are severe congenital birth malformations in the brain and spinal cord, which are caused by incomplete neural tube closure. They occur in 5.3 per 10,000 live births in the United States (1), and in >10/1,000 pregnancies in certain regions of China (2). Although the pathological mechanisms responsible for NTDs remain to be elucidated, at least 240 gene mutations in mice are known to increase the risk of NTDs, including several mutations in apoptosis-related genes (3).

Retinoids, a group of natural and synthetic derivatives of vitamin A, are important in the regulation of cellular proliferation, differentiation and apoptosis. Cell proliferation, differentiation, migration and apoptosis are key cellular processes in the regulation of neural tube closure (4). Vitamin $\mathrm{A}$ is required for central nervous system (CNS) development; a lack of vitamin A can result in the posterior hindbrain failing to develop as the cells destined to form this region of the CNS in the early embryo die through apoptosis (5). Cell apoptosis is increased in the neuroepithelium of the lumbosacral neural tube of rat embryos with all-trans-retinoic acid (ATRA)-induced spina bifida aperta (6). The increased cell death in the tail bud may be important in the formation of ATRA-induced spina bifida aperta (7).

Covalent histone modifications, including the methylation and acetylation of certain amino acid residues in chromatin histones, have been shown to be essential in gene regulation by modulating chromatin structure. Histone H3K27 acetylation (H3K27ac) is commonly located in gene-regulatory regions and is associated with gene activation, whereas trimethylated H3K27 (H3K27me3) represses transcription (8). Rada-Iglesias et al (9), showed that H3K27ac at specific differentiation gene enhancer regions is necessary for the conversion of human embryonic stem cells (hESCs) into neural stem cells. Balmer et al (10) used the differentiation of hESCs into neuroectodermal precursors as a model to investigate the mechanism underlying the effect of the histone deacetylase inhibitor trichostatin A, which was found to cause epigenetic changes and disturbed neural development. Mutations in genes encoding histone deacetylases, including EP300, CREBBP and CITED2, have also been shown to cause NTDs (11). In 
addition, our previous study demonstrated abnormal epigenetic modifications in human fetuses with NTDs $(12,13)$, although the detailed mechanisms and target genes remain to be elucidated. The present study investigated the association between epigenetic modifications and the expression of apoptosis-related genes in NTDs.

\section{Materials and methods}

Animals. All animal experiments were performed in accordance with the standards of the Capital Institute of Pediatrics Ethics Committee (Beijing, China; permit no: DWLL2015015). Adult male and female C57BL/6J mice were placed together at 08:00 a.m. for timed mating, and when a vaginal plug was observed at 10:00 a.m., this was considered as embryonic day 0 (E0). Subsequently, three doses of ATRA $(20 \mathrm{mg} / \mathrm{kg}$ body weight; Sigma; Merck KGaA, Darmstadt, Germany) suspended in olive oil were administered intraperitoneally into the mothers on gestational day (GD)8.0 at $0 \mathrm{~h}, \mathrm{GD} 8.0$ at $6 \mathrm{~h}$, and GD8.0 at $12 \mathrm{~h}$ when the neural tube and neuropore were fusing. The control consisted of olive oil administration, and mice were treated with the same volume of pure olive oil at the same time points as a control group, as described previously (14). E18.0 fetuses were delivered by cesarean section and 18 brain tissue samples from the fetuses with NTDs were obtained and pooled into three groups. At the same time, 18 brain tissue samples from 18 normal fetuses were pooled randomly to create three control groups.

$R N A$ extraction and reverse transcription-quantitative polymerase chain reaction ( $R T-q P C R$ ) analysis. Total RNA was isolated from tissue samples using TRIzol (cat. no. 15596026; Invitrogen; Thermo Fisher Scientific, Inc., Waltham, MA, USA) and analyzed on an Applied Biosystems 7500 Real-Time PCR system(AppliedBiosystems; ThermoFisher Scientific,Inc.) using a first strand cDNA synthesis kit (K1612; TransGen Biotech Co., Ltd., Beijing, China). Amplification was performed according to the following conditions: 1 cycle at $95^{\circ} \mathrm{C}$ for $10 \mathrm{~min}, 40$ cycles at $95^{\circ} \mathrm{C}$ for $15 \mathrm{sec}$ and $60^{\circ} \mathrm{C}$ for $1 \mathrm{~min}$. The apoptosis-related genes were detected using the Mouse Apoptosis PCR Array from Qiagen GmbH (Hilden Germany; http://www.sabiosciences .com/rt_pcr_product/HTML/PAMM-012Z.html) according to the manufacturer's protocols. The Mouse Apoptosis PCR Array was used to profile the expression of 84 key genes involved in programmed cell death. This array includes tumor necrosis factor (TNF) ligands and their receptors, members of the B-cell lymphoma 2 (bcl-2), caspase, inhibitor of apoptosis (IAP), TNF receptor-associated factor, caspase activator of recruitment domain, death domain, death effector domain, and cell death-inducing DFFA-like effector families, in addition to genes involved in the p53 and DNA damage pathways. All the apoptosis-related genes are listed in Table I. The data were analyzed using Qiagen RT ${ }^{2}$ Profiler PCR Array Data Analysis version 3.5 (Qiagen GmbH; product. no. 330231; cat. no. PAMM-012Z). The expression levels of target genes were normalized to $\beta$-actin and $\beta 2$ microglobulin. Fold changes were determined using the $2^{-\Delta \Delta C q}$ method (15).

Chromatin immunoprecipitation (ChIP) assays. The cross-linked, sonicated DNA products were incubated with
H3K27ac (cat. no. 17-683; Abcam, Cambridge, UK) and H3K27me3 (cat. no. 17-622; EMD Millipore, Billerica, MA, USA) antibodies, with mouse IgG antibody as a negative control. ChIP (cat. no. 49-2024; Invitrogen; Thermo Fisher Scientific, Inc.) and RT-qPCR analysis was performed using the ABI 7500 system. All primers used for ChIP-PCR are listed in Table II. Relative enrichment of histone modification was determined as follows: $2^{\text {(input-Cq) }}{ }_{\text {NTDs }} / 2^{\text {(input-Cq) }}$ control.

Pathway analysis. To investigate the possible biological interactions of the differentially regulated genes using GNCPro (http://gncpro.sabiosciences.com/gncpro.gncpro. php), an online software tool developed and maintained by SABiosciences (Qiagen $\mathrm{GmbH}$, Hilden, Germany) for collating gene and pathway interactions.

Gene mapping in the genome. All TNF-related gene sets were mapped in the genome of mouse using the RefSeq track of the University of California-Santa Cruz (UCSC) Genome Browser (version NCBI37/mm9; July 2007), Histone H3K27ac and H3K27me3 ChIP-seq profiles of the mouse brain were analyzed together. ChIP-seq data is presented as the number of reads overlapping genomic windows.

Statistical analysis. The transcript and histone modification enrichment analyses were performed $\geq 3$ times and the results were compared between the experimental and control groups using Student's t-tests. $\mathrm{P}<0.05$ was considered to indicate a statistically significant difference. Statistical analysis was performed using SPSS for Windows, v13.0 (SPSS Inc., Chicago, IL, USA).

\section{Results}

Changes in the transcription of apoptosis-related genes in NTD mice. To detect changes in the expression of apoptosis-related genes during the development of RA-induced NTDs, the present study examined the mRNA expression levels of 84 key apoptosis-related genes in brain tissues obtained from E18 fetuses. According to the gene expression profiles, the three NTD samples were clustered into one group, whereas the three normal control samples were clustered into another group, indicating that the transcription levels of these genes differed significantly between the NTD and control mice (Fig. 1A). Among the 84 key genes involved in programmed cell death, 13 were downregulated and one was upregulated in the NTD group, compared with the control (Fig. 1B). The downregulated genes were Tnf, annexin A5 (Anxa5), apoptosis inhibitor 5 (Api5), Bcl2-associated athanogene 3 (Bag3), baculoviral IAP repeat-containing 3 (Birc3), caspase (Casp)12, Casp4, Casp8, lymphotoxin $\beta$ receptor ( $L t b r)$, NLR family, apoptosis inhibitory protein 2 (Naip2), TNF receptor superfamily (Tnfrsf) la, Tnfsf10 and Tnfsf12, whereas the single upregulated gene was nucleolar protein 13 (Nol3), which belongs to the IAP family (Fig. 1C).

Subsequently, analysis was performed for candidate genes using GNCPro online. The genes were classified as anti-apoptotic or pro-apoptotic regulators. There was a significant TNF-related network involved in NTD formation (Fig. 2). The Tnf, Ltbr, Tnfrsfla, Tnfsf10 and Tnfsf12 genes all 
Table I. Functional gene grouping of the 84 key apoptosis-related genes.

Function

Genes

Induction of apoptosis Induction of apoptosis DNA damage

Extracellular signals Other

Regulation of apoptosis Negative regulation

Positive regulation

Caspases and regulators Caspases

Caspase activators

Caspase inhibitors

Anti-apoptotic

DEATH domain proteins
Cradd, Fadd, Tnf, Tnfrsflob (Dr5)

Cidea, Cideb, Trp53, Trp63, Trp73

Cflar (Casper), Dapk1

Bad, Bak1, Bax, Bcl10, Bcl2l11, Bid, Bnip3, Bok, Casp1 (Ice), Casp12, Casp14, Casp2, Casp3, Casp4, Casp6, Casp8, Cd70 (Tnfsf7), Dffa, Dffb, Diablo (Smac), Fas (Tnfrsf6), Fas1 (Tnfsf6), Mapk1 (Erk2), Nod1 (Card4), Pycard (Tms1/Asc), Tnfsf10 (Trail), Trp53bp2, Traf3

Bag1, Bag3, Bcl10, Bcl2, Bcl2ala, Bcl2l2 (Bcl-X), Bcl2l10, Bcl2l2, Birc2 (C-Iap2), Bir3 (C-Iap1), Bnip2, Bnip3, Bnip3l, Casp3, Cd40lg (Tnfsf5), Nol3, Polb, Prdx2, Tnf, Trp63, Xiap (Birc4)

Abl1, Akt1, Anxa5, Bad, Bak1, Bax, Bcl2l11, Bid, Bnip3, Bnip3l, Card10, Casp1 (Ice), Casp14, Casp2, Casp4, Casp6, Casp8, Cd40 (Tnfrsf5), Cd70 (Tnfsf7), Cideb, Cradd, Fadd, Fasl (Tnfsf6), Gadd45a, Ltbr, Nod1 (Card4), Pycard (Tms1/Acs), Tnf, Tnfrsf10b (Dr5), Tnfsf10 (Trail), Tnfsf12, Trp53, Trp53bp2, Traf1, Traf2, Traf3

Casp1 (Ice), Casp12, Casp14, Casp2, Casp3, Casp4, Casp6, Casp7, Casp8, Casp9, Cflar (Casper), Cradd, Pycard (Tms1/Asc)

Aifml (Pdcd8), Apaf1, Bax, Bcl2l10, Caspl (Ice), Casp9, Nod1 (Card4), Pycard (Tms1/Asc), Tnfrsf10B (Dr5), Trp53

Birc5, Xiap (Birc4)

Akt1, Api5, Atf5, Bag1, Bag3, Bax, Bcl2, Bcl2l1 (Bcl-x), Bcl2l10, Bcl2l2, Birc5, Bnip2, Bnip3, Bnip3l, Cd40lg (Tnfsf5), Cflar (Casper), Dad1, Dapk1, Fas (Tnfrsf6), Igf1R, Il10, Lhx4, Mcl1, Naipl (Birc1), Naip2, Nfkb1, Nme5, Nol3, Polb, Prdx2, Tnf, Trp63, Xiap (Birc4)

Cradd, Dapk1, Fadd, Nfkb1, Ripk1, Tnfrsf10b (Dr5), Tnfrsf11b, Tnfrsfla
Table II. Primer sequences for chromatin immunoprecipitation assay.

\begin{tabular}{lll}
\hline Gene & Primer & \multicolumn{1}{c}{ Primer sequence (5'-3') } \\
\hline Tnf & Forward & CCATGCGTCCAGCTGACTAA \\
& Reverse & TCCCCTGGCCACATCTTTC \\
Tnfsf12 & Forward & GAGTAGGTGTGGATGCTGCGT \\
& Reverse & GTCTCCCTGCACACACCTCA \\
Tnfsf10 & Forward & AGTGGCCGTGTGCTCTCAG \\
& Reverse & TCTGCACTTGGGAAAGGCAT \\
Tnfsf1a & Forward & GAGGGTGGGTTGCTAGCGA \\
& Reverse & AGCAAACCTGGCGTCAAACTA \\
Ltbr & Forward & TTTGAAGAGGTAGCGGCATGT \\
& Reverse & GCCTCTCCTTGGTCTCTTTGTC \\
Casp8 & Forward & CCATAGCAGGCCATTTAGCTG \\
& Reverse & ATGCAGGCTGCTGGAGAAA \\
Casp3 & Forward & GCAAGACAGCTCACCCTGAA \\
& Reverse & TTCCCATTGCCTAAAGCCGA \\
Nfkb1 & Forward & GTGGCGCTGAAGGACTGTAT \\
& Reverse & TCTACAGCACAGCACCGAAT \\
\end{tabular}

Tnf, tumor necrosis factor; Tnfsf, Tnf superfamily; Ltbr, lymphotoxin $\beta$ receptor; Casp, caspase; Nfkb1, nuclear factor- $\kappa \mathrm{B}$ subunit b1. encode TNF superfamily or TNF-related apoptosis-inducing ligand (TRAIL) superfamily proteins. The activation of these proteins by their associated ligands rapidly activates caspase- 8 and leads to cell destruction (16,17). Casp12, Casp4 and Casp8 genes encode caspase family proteins, which are key proteins regulating apoptosis. Casp8-knockout mice show abnormal yolk sac vasculature and various defects in the developing heart and neural tube $(18,19)$. The proteins encoded by Birc3, Naip2 and Nol3 belong to the IAP family, the members of which bind and inhibit caspases, and are important regulators of cell differentiation and cell death (20). The functions of Anxa5 and Bag3 remain to be elucidated. These data indicated that the transcription levels of certain apoptosis-related genes were abnormally activated in RA-induced NTDs.

Abnormal histone modifications in TNF-related gene promoters. To determine the mechanisms responsible for the ectopic changes in TNF-related gene expression, the present study examined the histone-modification status in the regulatory regions of the altered genes. H3K27ac and $\mathrm{H} 3 \mathrm{~K} 27 \mathrm{me} 3$ profiles were analyzed according to the current UCSC database (July 2007; NCBI37/mm9). The H3K27ac and H3K27me3 modifications were all targeted to the regulatory region of TNF-related genes (Tnf, Tnfrsfla, Tnfsf10, Tnfsf12 and Casp8) in the brain (Fig. 3A-E). All of these TNF-related genes were regulated by $\mathrm{p} 300$, which functions as a histone 


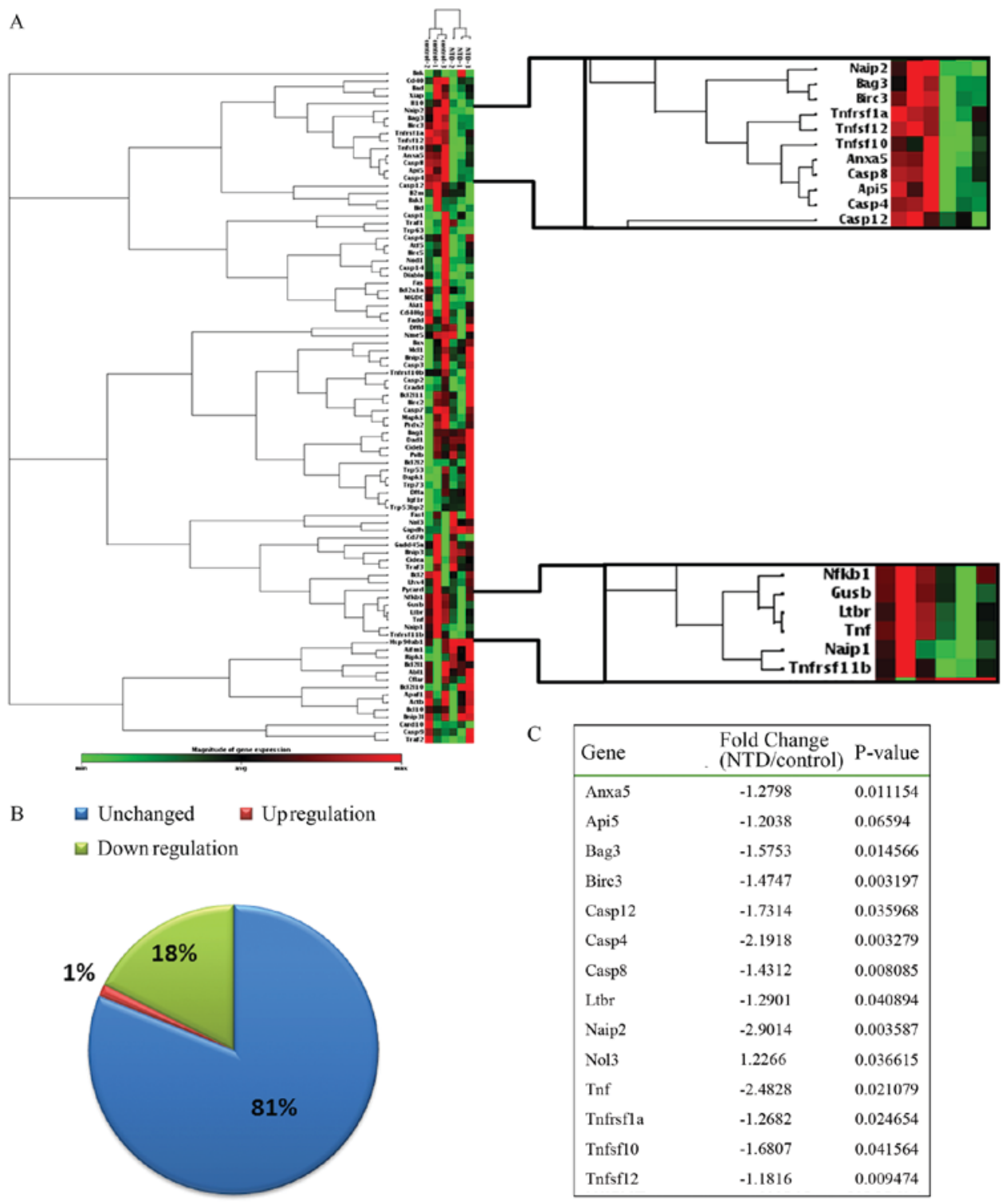

Figure 1. Apoptosis-related gene expression profiles of retinoic acid-induced NTD and normal mouse brains. (A) Cluster analysis of data from the mouse apoptosis polymerase chain reaction array. Red indicates upregulation and green indicates downregulation. (B) Summary of expression profile analysis of the 84 key genes involved in programmed cell death. (C) Differentially expressed genes. NTD, neural tube defect.

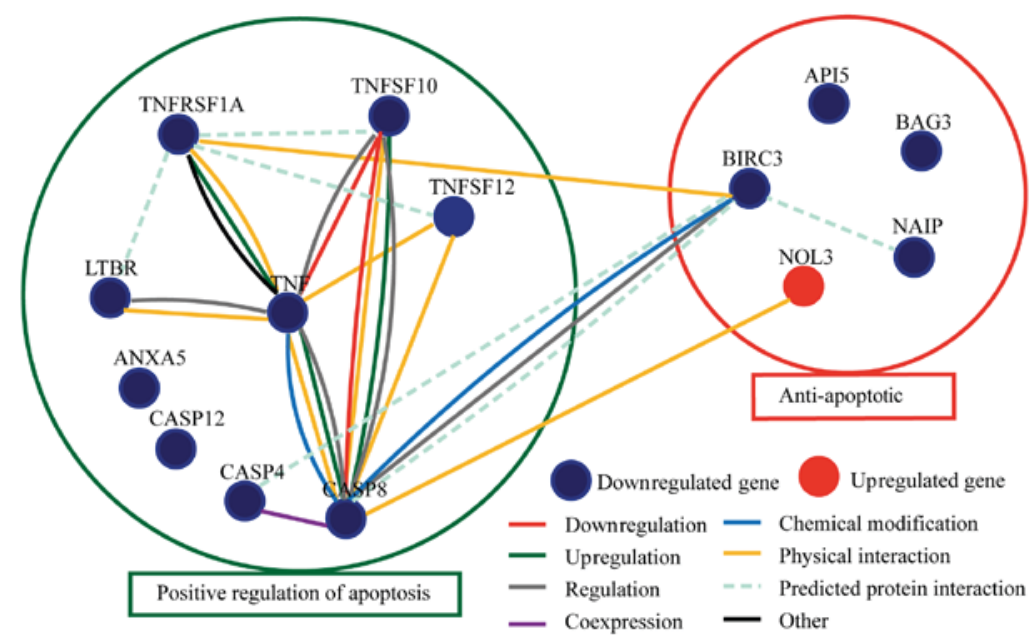

Figure 2. Interaction analysis of differentially expressed genes. The gene expression profile of the NTD mice differed from that of the normal controls. One gene was upregulated and 13 were downregulated in the NTD mice. The TNF pathway was the pathway with the highest representation among the differentially expressed genes. NTD, neural tube defect. 
acetyltransferase and regulates transcription via chromatin remodeling. A search was performed of the regulatory regions of TNF-related genes in the brain using the Genome Browser, and it was found that the TNF-related genes were also bound to $\mathrm{p} 300$, which indicated that these genes were regulated by histone acetylransferase P300 (Fig. 3). A ChIP-qPCR primer was designed from the $1 \mathrm{~kb}$ relative to the transcription start site in the highly targeted region, to determine whether the altered expression of apoptosis-related genes was caused by abnormal regulation of $\mathrm{H} 3 \mathrm{~K} 27 \mathrm{ac}$ and $\mathrm{H} 3 \mathrm{~K} 27 \mathrm{me} 3$.

According to the results of the ChIP analysis, H3K27ac bound to the regulatory regions of differentially regulated (Tnf, Casp8, Ltbr, Tnfrsfla, Tnfsf10 and Tnfsf12) and non-differentially regulated genes, Casp3 and nuclear factor $(\mathrm{NF})-\kappa \mathrm{B}$ subunit $1(N f k b l)$, in the normal control mice (Fig. 4). However, H3K27ac-binding to the differentially regulated genes was markedly decreased in the NTD mice (Fig. 4), whereas binding to the unchanged genes Casp3 and $N f k b l$ was unaffected. There was no significant difference in the binding of $\mathrm{H} 3 \mathrm{~K} 27 \mathrm{me} 3$ to apoptosis-related genes between the NTD and normal control mice. These results suggested that the downregulated expression of TNF-related genes in the RA-induced NTD mice may have been caused by abnormal $\mathrm{H} 3 \mathrm{~K} 27 \mathrm{ac}$ binding in their regulatory regions; this confirmed the aberrant epigenetic control of these genes in NTDs.

\section{Discussion}

NTDs result from the failure of complete neurulation during the fourth week of embryogenesis. Neurulation is a complex and multistep process, involving the precise temporal and spatial regulation of gene expression (21-23). Cell apoptosis involves a process of autonomic ordered programmed cell death, which is essential for maintaining homeostasis. Apoptosis is crucial in vital biological processes, including normal embryonic development, cell signaling, the immune response, and cell death (24). Apoptosis is also important in the morphogenesis and homeostasis of the developing CNS through its involvement in the folding, pinching off and fusion of neural walls, in neural precursor selection, and in the postmitotic competition of neurons for their cellular targets (25-27). The inhibition of caspase activity in chickens prevents neural tube closure, suggesting that apoptosis is required for neurulation, whereas reduced or excessive apoptosis is associated with the failure of neural tube closure in mutant mice (28). Caspases controlling apoptosis have also been shown to be involved in metazoan embryogenesis and homeostasis (29). Previous studies have revealed that apoptosis occurs during normal development of the CNS (30). Two fundamentally different mechanisms involving apoptosis-related genes can lead to NTDs. In the 129/SvJ genetic background, apoptotic peptidase activating factor 1 (apaf-1)-, casp-9-, or casp-3-knockout can cause neurodevelopmental abnormalities. A deficiency of proapoptotic regulators in these mutants leads to reduced apoptosis, resulting in an excess of neuroepithelial cells in the hindbrain, midbrain and forebrain, and subsequent gross neuronal disorders (31-34). This suggests that apoptosis is the primary mechanism whereby mammals prevent the survival of excess cells in the developing nervous system. By contrast, NTDs can also occur as a result of excessive cell death,

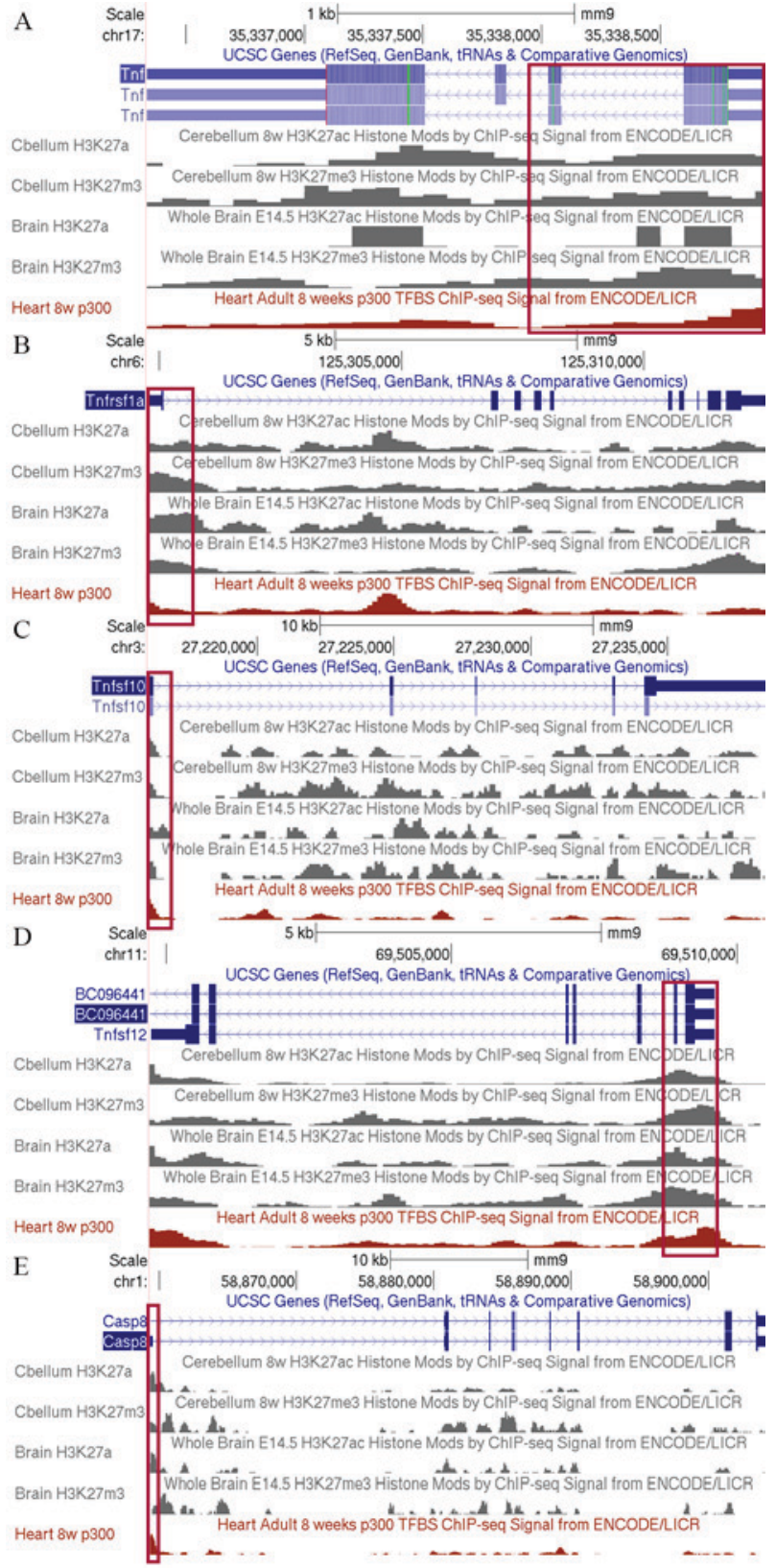

Figure 3. Histone $\mathrm{H} 3 \mathrm{~K} 27 \mathrm{ac}$ and $\mathrm{H} 3 \mathrm{~K} 27 \mathrm{me} 3$ enrichment profiles of TNF-related genes in the mouse brain. Screenshots from the UCSC genome browser showing H3K27ac and H3K27me3 ChIP-seq data of the mouse brain. ChIP-seq data is presented as the number of reads overlapping genomic windows. There was a high association between $\mathrm{H} 3 \mathrm{~K} 27 \mathrm{ac}$ and $\mathrm{H} 3 \mathrm{~K} 27 \mathrm{me} 3$ modification in the 1,000 bp downstream of the expression of TNF-related genes in the mice. The red boxes indicate the region $1 \mathrm{~kb}$ downstream from transcription start site. (A) Tnf, (B) Tnfrsfla, (C) Tnfsf10, (D) Tnfsf12, (E) Casp8. TNF, tumor necrosis factor; ChIP, chromatin immunoprecipitation; H3K27ac, H3K27 acetylation; H3K27me3, trimethylated H3K27.

leaving insufficient cells for neural tube closure. A previous study showed that genetic defects in $\mathrm{Bcl}-10$ and inhibitor of $\mathrm{NF}-\kappa \mathrm{B}$ kinase, which activate $\mathrm{NF}-\kappa \mathrm{B}$ in the anti-apoptotic pathway, cause exencephaly during neurulation (31-34). The activation of $\mathrm{NF}-\kappa \mathrm{B}$ in vivo is important in protecting neurons against excessive apoptosis during development. These apoptosis-related genes may be involved in NTD formation via the positive regulation of neuronal survival. Therefore, inhibited and increased apoptosis can cause NTDs and increase the risk of exencephaly. 


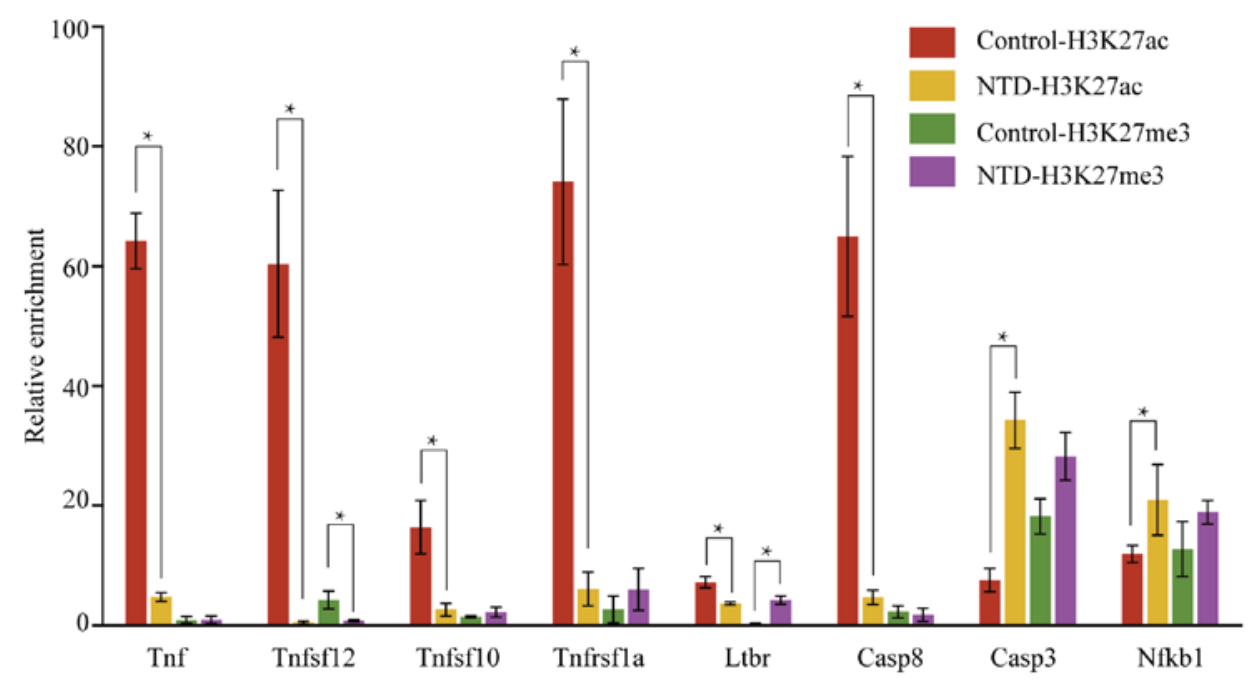

Figure 4. Chromatin immunoprecipitation assay of H3K27ac and H3K27me3 binding to TNF-related genes in the normal and NTD fetal brains. Results for all TNF-related differentially expressed and two non-differentially expressed (casp3 and $\mathrm{Nfkb1}$ ) genes. In the TNF-related gene promoter, H3K27ac enrichment was markedly downregulated in the NTD group. "P<0.05. NTD, neural tube defect; TNF, tumor necrosis factor; H3K27ac, H3K27 acetylation; H3K27me3, trimethylated H3K27.

Retinoids, a group of natural and synthetic derivatives of vitamin A, have important regulatory roles in cellular proliferation, differentiation and apoptosis. The results of the present study indicated that pro- and anti-apoptotic genes were abnormally expressed, suggesting that the two mechanisms are involved in RA-induced NTD formation in mice. Rodriguez-Leon et al revealed a mechanism by which RA, in conjunction with bone morphogenetic proteins, may regulate programmed cell death (35). Following ATRA treatment, Boutzen et al found that the reduction of cell viability and the induction of apoptosis led to a specific and marked reduction in the size and number of L-CFUs (36). Apoptosis can be affected by RA in certain developmental systems, including the neural crest cells of the rhombencephalic segments (37). Sulik and Dehart (38) investigated limb malformations, and it was found that RA resulted in excessive cell death in regions of programmed cell death and led to subsequent malformations of the affected regions. Liu et al (39) exposed the human K562 myeloid leukemia cell line to ATRA, which led to apoptosis increasing significantly. ATRA also can exert anti-apoptotic effects through the myeloid cell leukemia 1 protein at the transcriptional level (40).

The results of the present showed that TNF family genes, including Tnf, Tnfrsfla, Tnfsf10, Tnfsf 12 and Ltbr and Casp8, were involved in NTD formation. Activation by their associated ligands rapidly activates caspase- 8 and leads to cell death $(17,18)$. Casp 8 has been shown to have dual roles in the activation of apoptosis and suppression of necrosis, which are two important processes in mouse embryonic development (41). Previous studies have focused on the critical role of intrinsic apoptosis in mammalian neural tube development, whereas the present study demonstrated that extrinsic-apoptosis-related genes, including the Casp 8 gene, were also aberrantly expressed in RA-induced NTD.

The results of the present study demonstrated that the expression levels of TNF-related genes were decreased as a result of the abnormal regulation of H3K27ac. H3K27ac is associated with transcription activation $(41,42)$; therefore, decreased H3K27ac binding to the gene regulatory region results in the downregulation of gene expression.

A change between $\mathrm{H} 3 \mathrm{~K} 27 \mathrm{me} 3$ and $\mathrm{H} 3 \mathrm{~K} 27 \mathrm{ac}$ at specific differentiation gene enhancer regions has been shown to be essential for the conversion of hESCs into neural stem cells (9). Hayashi-Takanaka et al (43) monitored the levels of H3K27ac in mouse preimplantation embryos produced by in vitro fertilization and somatic cell nuclear transfer, which revealed that a high level of $\mathrm{H} 3 \mathrm{~K} 27 \mathrm{ac}$ was required for normal embryonic development. Overall, these findings indicate that abnormalities in $\mathrm{H} 3 \mathrm{~K} 27 \mathrm{ac}$ are involved in the etiology of NTDs, including via the downregulation of apoptosis-related genes.

In conclusion, the present study demonstrated that apoptosis-related gene expression levels were altered in NTDs, possibly as a result of epigenetic modifications involving H3K27ac. Therefore, aberrant histone modifications during early pregnancy may contribute to the formation of NTDs by affecting neural development. Further investigations are required to confirm these results.

\section{Acknowledgements}

The authors would like to thank all participating hospitals and medical staff for their assistance with sample collection and recording of clinical information.

\section{Funding}

This study was supported by the National Natural Science Foundation of China, Beijing, China (grant nos. 81741044, and 81471163) and the National '973' project (grant no. 2013CB945404).

\section{Availability of data and materials}

The analyzed data sets generated during the study are available from the corresponding author on reasonable request. 


\section{Authors' contributions}

QZ and HL initiated and conceived the study; QZ, HL and CW designed and performed the experiments; QZ, XL and CW wrote the manuscript; BB performed animal models; XL and $\mathrm{HC}$ verified the changes of genes; $\mathrm{CW}$ and XL performed the bioinformatics and biostatistics analysis under the guidance of QZ. All authors read and approved the final manuscript.

\section{Ethics approval and consent to participate}

All animal experiments were performed in accordance with the standards of the Capital Institute of Pediatrics Ethics Committee (Beijing, China; permit no: DWLL2015015).

\section{Consent for publication}

Not applicable.

\section{Competing interests}

The authors declare that they have no competing interests.

\section{References}

1. Williams J, Mai CT, Mulinare J, Isenburg J, Flood TJ, Ethen M, Frohnert B and Kirby RS; Centers for Disease Control and Prevention: Updated estimates of neural tube defects prevented by mandatory folic Acid fortification-United States, 1995-2011. MMWR Morb Mortal Wkly Rep 64: 1-5, 2015.

2. Li Z, Ren A, Zhang L, Ye R, Li S, Zheng J, Hong S, Wang T and Li Z: Extremely high prevalence of neural tube defects in a 4-county area in Shanxi Province, China. Birth Defects Res A Clin Mol Teratol 76: 237-240, 2006.

3. Hansler A, Chen Q, Gray JD, Ross ME, Finnell RH and Gross SS: Untargeted metabolite profiling of murine embryos to reveal metabolic perturbations associated with neural tube closure defects. Birth Defects Res A Clin Mol Teratol 100: 623-632, 2014.

4. Copp AJ, Greene ND and Murdoch JN: The genetic basis of mammalian neurulation. Nat Rev Genet 4: 784-793, 2003.

5. Maden M, Gale E and Zile M: The role of vitamin A in the development of the central nervous system. J Nutr 128 (2 Suppl): 471S-475S, 1998.

6. Wei X, Li H, Miao J, Zhou F, Liu B, Wu D, Li S, Wang L, Fan Y, Wang W and Yuan Z: Disturbed apoptosis and cell proliferation in developing neuroepithelium of lumbo-sacral neural tubes in retinoic acid-induced spina bifida aperta in rat. Int $\mathbf{J}$ Dev Neurosci 30: 375-381, 2012.

7. Alles AJ and Sulik KK: Retinoic acid-induced spina bifida: Evidence for a pathogenetic mechanism. Development 108: 73-81, 1990.

8. Ha M, Ng DW, Li WH and Chen ZJ: Coordinated histone modifications are associated with gene expression variation within and between species. Genome Res 21: 590-598, 2011.

9. Rada-Iglesias A, Bajpai R, Swigut T, Brugmann SA, Flynn RA and Wysocka J: A unique chromatin signature uncovers early developmental enhancers in humans. Nature 470: 279-283, 2011.

10. Balmer NV, Weng MK, Zimmer B, Ivanova VN, Chambers SM, Nikolaeva E, Jagtap S, Sachinidis A, Hescheler J, Waldmann T and Leist M: Epigenetic changes and disturbed neural development in a human embryonic stem cell-based model relating to the fetal valproate syndrome. Hum Mol Genet 21: 4104-4114, 2012.

11. Copp AJ and Greene ND: Genetics and development of neural tube defects. J Pathol 220: 217-230, 2010.

12. Wang L, Wang F, Guan J, Le J, Wu L, Zou J, Zhao H, Pei L, Zheng $X$ and Zhang T: Relation between hypomethylation of long interspersed nucleotide elements and risk of neural tube defects. Am J Clin Nutr 91: 1359-1367, 2010.
13. Zhang Q, Xue P, Li H, Bao Y, Wu L, Chang S, Niu B, Yang F and Zhang T: Histone modification mapping in human brain reveals aberrant expression of histone $\mathrm{H} 3$ lysine 79 dimethylation in neural tube defects. Neurobiol Dis 54: 404-413, 2013.

14. Chen S, Bai B, Wang X, Li H and Zhang T: Retinoic acid induced neural tube defects in C57 mice in a dose-dependent and time-specific way. Chin J Birth Health Heredity: 126-128, 2014 (In Chinese).

15. Livak KJ and Schmittgen TD: Analysis of relative gene expression data using real-time quantitative PCR and the 2(-Delta Delta C(T)) method. Methods 25: 402-408, 2001.

16. Martin DA, Siegel RM, Zheng L and Lenardo MJ: Membrane oligomerization and cleavage activates the caspase- 8 (FLICE/MACHalpha1) death signal. J Biol Chem 273: 4345-4349, 1998.

17. Mahmood Z and Shukla Y: Death receptors: Targets for cancer therapy. Exp Cell Res 316: 887-899, 2010.

18. Varfolomeev EE, Schuchmann M, Luria V, Chiannilkulchai N, Beckmann JS, Mett IL, Rebrikov D, Brodianski VM, Kemper OC, Kollet $\mathrm{O}$, et al: Targeted disruption of the mouse Caspase 8 gene ablates cell death induction by the TNF receptors, Fas/Apol, and DR3 and is lethal prenatally. Immunity 9: 267-276, 1998.

19. Sakamaki K, Inoue T, Asano M, Sudo K, Kazama H, Sakagami J, Sakata S, Ozaki M, Nakamura S, Toyokuni S, et al: Ex vivo whole-embryo culture of caspase-8-deficient embryos normalize their aberrant phenotypes in the developing neural tube and heart. Cell Death Differ 9: 1196-1206, 2002.

20. Dubrez-Daloz L, Dupoux A and Cartier J: IAPs: More than just inhibitors of apoptosis proteins. Cell Cycle 7: 1036-1046, 2008.

21. Tanabe Y and Jessell TM: Diversity and pattern in the developing spinal cord. Science 274: 1115-1123, 1996.

22. Smith JL and Schoenwolf GC: Neurulation: Coming to closure. Trends Neurosci 20: 510-517, 1997.

23. Colas JF and Schoenwolf GC: Towards a cellular and molecular understanding of neurulation. Dev Dyn 221: 117-145, 2001.

24. Sherif ZA: Caspase activation and aberrant cell growth in a p53(+/+) cell line from a Li-Fraumeni syndrome family. Genet Res Int 2015: 789201, 2015.

25. Nicotera P: Development and death of neurons: Sealed by a common fate? Cell Death Differ 9: 1277-1278, 2002.

26. Hidalgo A and Ffrench-Constant C: The control of cell number during central nervous system development in flies and mice. Mech Dev 120: 1311-1325, 2003.

27. De Zio D, Giunta L, Corvaro M, Ferraro E and Cecconi F: Expanding roles of programmed cell death in mammalian neurodevelopment. Semin Cell Dev Biol 16: 281-294, 2005.

28. Massa V, Savery D, Ybot-Gonzalez P, Ferraro E, Rongvaux A, Cecconi F, Flavell R, Greene ND and Copp AJ: Apoptosis is not required for mammalian neural tube closure. Proc Natl Acad Sci USA 106: 8233-8238, 2009.

29. Salvesen GS and Ashkenazi A: Snapshot: Caspases. Cell 147: 476.e1, 2011.

30. Naruse I and Keino H: Apoptosis in the developing CNS. Prog Neurobiol 47: 135-155, 1995.

31. Kuida K, Haydar TF, Kuan CY, Gu Y, Taya C, Karasuyama H, Su MS, Rakic P and Flavell RA: Reduced apoptosis and cytochrome c-mediated caspase activation in mice lacking caspase 9. Cell 94: 325-337, 1998.

32. Kuida K, Zheng TS, Na S, Kuan C, Yang D, Karasuyama H, Rakic P and Flavell RA: Decreased apoptosis in the brain and premature lethality in CPP32-deficient mice. Nature 384: 368-372, 1996.

33. Cecconi F, Alvarez-Bolado G, Meyer BI, Roth KA and Gruss P: Apaf1 (CED-4 homolog) regulates programmed cell death in mammalian development. Cell 94: 727-737, 1998.

34. Hakem R, Hakem A, Duncan GS, Henderson JT, Woo M, Soengas MS, Elia A, de la Pompa JL, Kagi D, Khoo W, et al: Differential requirement for caspase 9 in apoptotic pathways in vivo. Cell 94: 339-352, 1998.

35. Rodriguez-Leon J, Merino R, Macias D, Gañan Y, Santesteban E and Hurle JM: Retinoic acid regulates programmed cell death through BMP signalling. Nat Cell Biol 1: 125-126, 1999.

36. Boutzen H, Saland E, Larrue C, de Toni F, Gales L, Castelli FA, Cathebas M, Zaghdoudi S, Stuani L, Kaoma T, et al: Isocitrate dehydrogenase 1 mutations prime the all-trans retinoic acid myeloid differentiation pathway in acute myeloid leukemia. J Exp Med 213: 483-497, 2016.

37. Graham A, Francis-West P, Brickell P and Lumsden A: The signalling molecule BMP4 mediates apoptosis in the rhombencephalic neural crest. Nature 372: 684-686, 1994. 
38. Sulik KK and Dehart DB: Retinoic-acid-induced limb malformations resulting from apical ectodermal ridge cell death. Teratology 37: 527-537, 1988

39. Liu WJ, Zhang T, Guo QL, Liu CY and Bai YQ: Effect of ATRA on the expression of HOXA5 gene in K562 cells and its relationship with cell cycle and apoptosis. Mol Med Rep 13: 4221-4228, 2016.

40. Holm KL, Indrevaer RL, Myklebust JH, Kolstad A, Moskaug JØ, Naderi EH and Blomhoff HK: Myeloid cell leukaemia 1 has a vital role in retinoic acid-mediated protection of Toll-like receptor 9 -stimulated B-cells from spontaneous and DNA damage-induced apoptosis. Immunology 149: 62-73, 2016.

41. Hnisz D, Abraham BJ, Lee TI, Lau A, Saint-André V, Sigova AA, Hoke HA and Young RA: Super-enhancers in the control of cell identity and disease. Cell 155: 934-947, 2013
42. Creyghton MP, Cheng AW, Welstead GG, Kooistra T, Carey BW, Steine EJ, Hanna J, Lodato MA, Frampton GM, Sharp PA, et al: Histone H3K27ac separates active from poised enhancers and predicts developmental state. Proc Natl Acad Sci USA 107: 21931-21936, 2010

43. Hayashi-Takanaka Y, Yamagata K, Wakayama T, Stasevich TJ, Kainuma T, Tsurimoto T, Tachibana M, Shinkai Y, Kurumizaka H, Nozaki $\mathrm{N}$ and Kimura $\mathrm{H}$ : Tracking epigenetic histone modifications in single cells using Fab-based live endogenous modification labeling. Nucleic Acids Res 39: 6475-6488, 2011.

This work is licensed under a Creative Commons Attribution-NonCommercial-NoDerivatives 4.0 International (CC BY-NC-ND 4.0) License. 